\title{
DISTRIBUCIÓN E INTENSIDAD DE LOS ESTUDIOS FAUNÍSTICOS SOBRE MARIPOSAS DIURNAS EN LA PENÍNSULA IBÉRICA E ISLAS BALEARES (LEPIDOPTERA, PAPILIONOIDEA Y HESPERIOIDEA)
}

\author{
H. Romo Benito* y E. García-Barros*
}

\begin{abstract}
RESUMEN
Se ha analizado la distribución actual de la intensidad de muestreo de las mariposas diurnas en la Península Ibérica e Islas Baleares, usando el sistema de proyección UTM, con una red de malla de cuadrículas de 10 y $50 \mathrm{~km}$ de lado. Se realizaron tres tipos de aproximaciones para valorar la cobertura geográfica de los datos, seleccionando el número de registros de la base de datos ATLAMAR1 como unidad de esfuerzo de muestreo: Una basada en criterios mínimos, otra en estimadores indirectos derivados de 25 cuadrículas UTM de $10 \mathrm{~km}$ de lado bien muestreadas, y una última mediante la utilización de curvas de acumulación que se aplicó a las cuadrículas de $50 \mathrm{~km}$ de lado.

Los resultados de todos los análisis coinciden en señalar un $6,8 \%$ de las cuadrículas de $10 \mathrm{~km}$ de lado y un $62,3 \%$ de las cuadrículas de $50 \mathrm{~km}$ como bien estudiadas. Aproximadamente un tercio del territorio se caracteriza por una notable escasez de registros de mariposas, principalmente algunas zonas de Galicia, gran parte de Castilla-La Mancha, y la zona interior de Andalucía.

Los resultados permiten disponer de una base sólida y objetiva en la que apoyar subsiguientes estudios sobre biodiversidad, biogeografía y conservación en relación con estos lepidópteros. Palabras clave: Mariposas, Lepidoptera, esfuerzo de muestreo, estudios faunísticos, curvas de acumulación, Península Ibérica, islas Baleares.
\end{abstract}

\section{ABSTRACT \\ Distribution and intensity of butterfly faunistic studies in the Iberian Peninsula and the Balearic Islands (Lepidoptera, Papilionoidea \& Hesperioidea)}

The distribution of the sampling effort of current faunistic data on the butterflies in the Iberian Peninsula and Balearic Islands has been analysed, using 10 and $50 \mathrm{~km}$ UTM grids. The number of records in the database ATLAMAR1 was selected as the sampling effort unit. Three approaches were attempted to evaluate the geographic coverage of the data: First, based on criteria of minimal conditions. Second, based on indirect estimators derived from $2510 \times 10 \mathrm{~km}$ UTM squares whose butterfly fauna is believed to be well sampled. Finally, accumulation curves were used to evaluate the completeness of faunistic knowledge in the $50 \mathrm{~km}$ UTM squares.

The different approaches were broadly coincident in indicating an adequate degree of knowledge for $6.8 \%$ of the $10 \times 10$ squares, and $62.3 \%$ of the $50 \times 50$ UTM squares. Approximately one third of the territory is characterised by a remarkable scarcity of butterfly records. This is especially true for some regions, namely Galicia, Castilla-La Mancha and inland Andalusia.

The results provide a reliable and objective basis for subsequent work on butterfly diversity, biogeography and conservation within the Iberian area.

Key words: Butterflies, Lepidoptera, sampling effort, faunistic studies, accumulation curves, Iberian Peninsula, Balearic Islands.

* Departamento de Biología (Zoología), Universidad Autónoma de Madrid. E-28049 Madrid, España. E-mail: helena.romo@uam.es 


\section{Introducción}

Las áreas mediterráneas constituyen uno de los puntos de mayor diversidad biológica en el mundo (Myers et al., 2000). Para poder determinar con criterios objetivos el interés conservacionista de especies y áreas geográficas, es necesario un conocimiento detallado de la distribución espacial de los organismos. Los parámetros que podrían servir para definir la conservación, como son entre otros la riqueza, la rareza o la endemicidad, podrían extrapolarse si se consiguen estimar estos diferentes parámetros que definen la diversidad de un grupo en las unidades geográficas bien muestreadas.

Los lepidópteros son uno de los grupos más diversos de insectos. Las mariposas diurnas (comprendidas en las superfamilias Papilionoidea y Hesperioidea) suelen ser consideradas entre los grupos de insectos más populares y mejor conocidos. Además, el carácter estenófago de sus larvas fitófagas hace suponer a estos insectos buenos indicadores de la calidad de conservación de los ecosistemas (New, 1991). La Península cuenta con una gran heterogeneidad de hábitats, y ocupa el tercer puesto en Europa, precedida de Italia y Francia, en cuanto a riqueza de especies (Kudrna, 2002).

Pero a pesar de la riqueza en especies de mariposas del territorio español (Munguira, 1995), es difícil actualmente decidir con cierto nivel de confianza qué zonas han sido adecuadamente prospectadas y cuáles no, algo trascendental a la hora de profundizar en el estudio de la distribución de la diversidad de especies de mariposas y sus causas (Martín \& Gurrea, 1990; Hortal et al., 2004). Esta estimación es importante, pues permite medir la fiabilidad de los inventarios biológicos y compararlos, facilitando la planificación de sucesivos muestreos, así como calcular el grado de esfuerzo que habría que llevar a cabo en una cuadrícula poco trabajada para llegar a un conocimiento óptimo de su fauna (referencias en Jiménez-Valverde \& Hortal, 2003). Hay antecedentes recientes de este tipo de estimación referidos a mariposas ibéricas, pero basados en un escaso volumen de registros $\mathrm{y}$ un número limitado de especies representativas (García-Barros \& Munguira, 1999; García-Barros et al., 2000), o bien tratando solo parte del territorio (por ejemplo García-Pereira, 2003; Hortal et al., 2004). Como consecuencia de la preparación de un atlas de distribución de estos insectos en el área ibero-balear (García-Barros et al., 2004), se ha acumulado una notable cantidad de información faunística que puede hoy emplearse para este propósito.
Hay varios métodos para comparar la densidad de esfuerzo en unidades geográficas de superficie homogénea, basados en la diversidad ecológica y estructura de las taxocenosis, las probabilidades de captura, y las curvas de acumulación o de colecta (Soberón \& Llorente, 1993; Gotelli \& Colwell, 2001). De acuerdo con diversos autores (Soberón \& Llorente, 1993; Hortal \& Lobo, 2002; JiménezValverde \& Hortal, 2003), la aproximación más eficaz para valorar la calidad de los inventarios se obtiene mediante las curvas de acumulación de especies, que relacionan el esfuerzo de muestreo con el número de especies encontrado. El cálculo es relativamente rápido para un número limitado de unidades de terreno, por ejemplo las cuadrículas UTM ibéricas de 50 ó $100 \mathrm{~km}$ de lado. Ahora bien, para un número elevado de unidades de área (varios miles, como es el caso de las cuadrículas de $10 \mathrm{~km}$ de lado del área de estudio), el cálculo de curvas de acumulación es largo y tedioso. Por este motivo, y dado que la información faunística sobre mariposas ibéricas se incrementa de modo más o menos regular en la actualidad, es tentador comprobar hasta qué punto algún cálculo estadístico sencillo derivado directamente de la información asociada a los registros (número de ejemplares, de colectores, fechas, etc., registrados en una cuadrícula) pudiera arrojar estimas de saturación comparables a las de una curva de acumulación. Esta aproximación no cuenta con un fundamento teórico particular (GarcíaBarros et al., 2000; Garcia-Pereira, 2003), sino que depende de la clase de información disponible, y de su distribución en una colección de datos concreta. Pero cuenta con la ventaja de la inmediatez de los resultados, especialmente si puede calibrarse por comparación con otros métodos. Por último, dependiendo de las características de la información faunística acumulada en una base de datos concreta, podría dudarse de que los registros como tales representen mejor medida de esfuerzo (como defienden Lobo \& Martín-Piera, 2002; Hortal \& Lobo, 2002) que, por ejemplo, el número de ejemplares. Por ello se creyó conveniente comprobar, en primer lugar, hasta qué punto el número de registros de la base de datos empleada representa la mejor medida del esfuerzo de muestreo.

Por lo tanto, este estudio se diseñó para cubrir los siguientes objetivos:

1) Comprobar si el número de registros es la mejor unidad para medir el esfuerzo de muestreo, comparando su correlación, y la de otras variables relacionadas (número de individuos, de fechas de muestreo, de publicaciones, o de colectores), con el número de especies censadas de cada cuadrícula. 
Tabla 1.- Se muestran las 25 coordenadas UTM de referencia, el número de registros que corresponden a cada una de ellas, el número de especies y ejemplares que poseen, el número de colectores diferentes, el número de años de los que se posee citas y el número de publicaciones en que se mencionan (ver Figura 1).

Table 1.- Description of the 25 UTM reference squares, their database-records, number of species and specimens, number of different collectors, number of different years for which we have data, and number of publications in which they are quoted (see Figure 1).

\begin{tabular}{|c|c|c|c|c|c|c|c|c|c|}
\hline Número & $\begin{array}{l}\text { Coordenada } \\
\text { UTM }\end{array}$ & $\begin{array}{l}\text { Localidad } \\
\text { principal }\end{array}$ & Provincia & $\begin{array}{c}\mathrm{N}^{0} \text { de } \\
\text { Registros }\end{array}$ & $\begin{array}{c}\mathrm{N}^{\mathrm{o}} \mathrm{de} \\
\text { especies }\end{array}$ & $\begin{array}{c}\mathrm{N}^{\mathbf{0}} \text { de } \\
\text { ejemplares }\end{array}$ & $\begin{array}{c}\mathrm{N}^{0} \text { de } \\
\text { colectores }\end{array}$ & $\begin{array}{l}N^{0} \text { de } \\
\text { años }\end{array}$ & $\begin{array}{c}\mathrm{N}^{0} \text { de } \\
\text { publicaciones }\end{array}$ \\
\hline 1 & $30 \mathrm{TXK} 37$ & Albarracín & Teruel & 2492 & 138 & 4354 & 79 & 61 & 94 \\
\hline 2 & 29SNC44 & Alcacer do Sal & Portugal & 158 & 46 & 172 & 11 & 12 & 11 \\
\hline 3 & 30 TVK43 & Aranjuez & Madrid & 849 & 73 & 1259 & 35 & 49 & 40 \\
\hline 4 & 31 TDF38 & Barcelona & Barcelona & 612 & 99 & 569 & 50 & 53 & 60 \\
\hline 5 & 31ТВН91 & Benasque (pueblo) & Huesca & 579 & 127 & 599 & 24 & 34 & 37 \\
\hline 6 & 29TPG01 & Boticas & Portugal & 299 & 80 & 299 & 4 & 16 & 6 \\
\hline 7 & $30 \mathrm{TVK} 37$ & Casa de Campo & Madrid & 2983 & 88 & 9524 & 70 & 55 & 56 \\
\hline 8 & 29TNE55 & Coimbra & Portugal & 198 & 48 & 198 & 12 & 14 & 10 \\
\hline 9 & 29SQA29 & Doñana (Palacio) & Huelva & 55 & 38 & 54 & 5 & 5 & 6 \\
\hline 10 & 30TWK 29 & Durón & Guadalajara & 1359 & 97 & 2911 & 6 & 24 & 16 \\
\hline 11 & 30TUN57 & Fuente De & Cantabria & 1064 & 132 & 1252 & 40 & 47 & 50 \\
\hline 12 & 30 STF 80 & Gibraltar & Cádiz & 682 & 75 & 926 & 37 & 29 & 49 \\
\hline 13 & 30TTK56 & Hervás & Cáceres & 797 & 99 & 949 & 27 & 33 & 25 \\
\hline 14 & 30TYN01 & Jaca & Huesca & 1134 & 123 & 1242 & 29 & 40 & 42 \\
\hline 15 & 30TVL12 & La Granja & Segovia & 1310 & 112 & 2640 & 45 & 40 & 50 \\
\hline 16 & 31TDG18 & La Molina & Gerona & 438 & 99 & 570 & 67 & 50 & 71 \\
\hline 17 & 29SNB20 & Lagos & Algarve & 240 & 41 & 252 & 12 & 12 & 13 \\
\hline 18 & 29SMC88 & Lisboa & Portugal & 626 & 64 & 1912 & 33 & 49 & 25 \\
\hline 19 & $30 \mathrm{TYN} 23$ & Panticosa & Huesca & 1431 & 132 & 1546 & 29 & 30 & 38 \\
\hline 20 & 31 TBF72 & Puertos de Tortosa & Tarragona & 517 & 87 & 685 & 19 & 30 & 34 \\
\hline 21 & 30SXG29 & Sierra Espuña & Murcia & 637 & 102 & 917 & 28 & 31 & 35 \\
\hline 22 & 30TWK96 & Tragacete & Cuenca & 1076 & 127 & 4841 & 23 & 19 & 37 \\
\hline 23 & 30TWL30 & Trillo & Guadalajara & 604 & 110 & 692 & 10 & 19 & 20 \\
\hline 24 & 30SVG60 & Veleta & Granada & 765 & 110 & 1522 & 58 & 46 & 66 \\
\hline 25 & 30TXM71 & Zaragoza (Capital) & Zaragoza & 189 & 60 & 199 & 9 & 28 & 13 \\
\hline
\end{tabular}

2) Mediante curvas de acumulación, evaluar la cobertura de los datos faunísticos en las cuadrículas de $50 \mathrm{~km}$ de lado de la Península e Islas Baleares.

3) Medir la cobertura de los datos mediante dos procedimientos diferentes a las curvas de acumulación o colecta, a dos escalas: cuadrículas de $10 \times 10 \mathrm{~km}$ y de 50 x $50 \mathrm{~km}$. Estos procedimientos comprenden a) criterios mínimos apoyados en pautas que sugieren que son cuadriculas fiables, y b) estimadores indirectos derivados de una muestra de 25 cuadriculas UTM diferentes de $10 \mathrm{~km}$ de lado bien muestreadas. Como consecuencia, es finalmente posible:

4) comparar los resultados de distintos métodos $y$

5) disponer de una primera evaluación de la cobertura geográfica de la información, en la escala de $10 \times 10 \mathrm{~km}$.

\section{Material y métodos}

DATOS, REPRESENTACIÓN GEOGRÁFICA, Y ANÁLISIS ESTADÍSTICO GENERAL. Este trabajo se ha realizado utilizando los 290.340 registros de mariposas diurnas de la Península Ibérica e islas Baleares que forman parte de la base de datos ATLAMARl (inédito; ver García-Barros et al., 2004). Los datos, relativos a 230 especies de lepidópteros pertenecientes a las superfamilias Papilionoidea y Hesperioidea, proceden de 1498 fuentes de información (bibliografía, colecciones, y otros), y datan del período comprendido entre 1784-2003. Cada registro incluye, entre otros datos, la especie, localidad o topónimo, día, mes y año de captura, colector, y fuente de información o referencia. Se ha considerado un registro diferente a otro cuando, para una misma especie, difiere en cualquiera de los demás campos. 


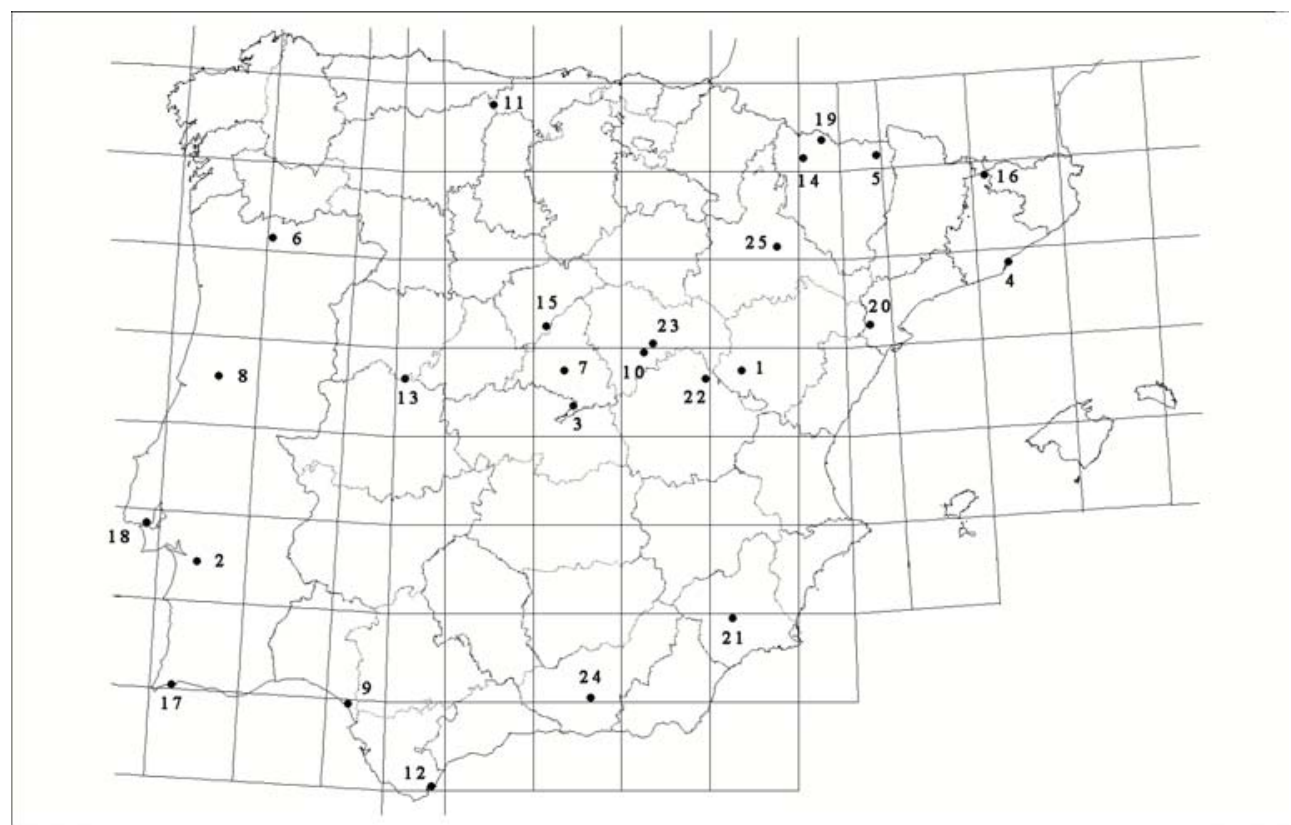

Fig. 1.- Localización de las 25 cuadrículas UTM de referencia de $10 \mathrm{~km}$ de lado en nuestro área de estudio (Península Ibérica e islas Baleares). Los números se corresponden con los de la Tabla 1.

Fig. 1.- Location of the 25 UTM reference $10 \times 10 \mathrm{~km}$ squares in the study area (the Iberian Peninsula and the Balearic Islands). Numbers correspond to those in Table 1.

Se utilizó el sistema de proyección UTM (Universal Transverse Mercator) con redes de malla de 10 y $50 \mathrm{~km}$ de lado. Las coordenadas UTM proporcionan unidades teóricamente de igual área en todas las latitudes comprendidas entre $0^{\circ}$ y $80^{\circ} \mathrm{N}$ y $\mathrm{S}$, y su flexibilidad de escalas permite transformar los datos a un tamaño de cuadrícula mayor o menor (Palomo \& Antúnez, 1992). Se ha estimado en 6395 el total de cuadrículas de $10 \mathrm{~km}$ de las que consta nuestro área de estudio, y en 281 para las cuadrículas de $50 \mathrm{~km}$. De ellas interesan un total de 4114 cuadrículas de $10 \mathrm{~km}$, y 277 de $50 \mathrm{~km}$ de lado, de las que se disponen datos. Para la realización de los mapas a escala de $10 \mathrm{~km}$ de lado se geocodificaron los datos utilizando el programa MapInfo (1994). En los mapas de $50 \mathrm{~km}$ las áreas de las zonas de compensación han sido tratadas como parte de las cuadrículas adyacentes a ellas, así como aquellas cuadrículas que presentan un porcentaje de tierra menor del 15\% (Figuras 4, 5 y 7 ).

Para el tratamiento de los datos se han utilizado los programas informáticos STATISTICA (Statsoft, 2004), EstimateS (Colwell, 2000) y SPSS (2003).

\section{ESTUDIO DE LA CALIDAD DE LOS INVENTARIOS}

1. Estimadores indirectos, cuadrículas de referencia y elección de la unidad de esfuerzo de muestreo. Se comprobó en primer lugar la correlación entre los valores acumulados de 9 variables para un porcentaje determinado de especies. Cada variable representa un porcentaje creciente de riqueza en cada cuadrícula. Se trata por tanto de calcular cuál representa mejor dicho porcentaje, para lo que se realizó una regresión múltiple por etapas hacia delante con el citado porcentaje de especies como variable dependiente, y como variables independientes el resto de las variables potencialmente representativas del esfuerzo de muestreo. Estas variables independientes son: 1- el número de ejemplares; 2- el número de localidades (topónimos diferentes en cada cuadrícula); 3- el número de fechas de muestreo (meses); 4- el número de colectores; 5- el número de fuentes (publicaciones donde han sido citados los datos); 6- el número de años; 7- el número de registros; 8el número de combinaciones diferentes de fecha de 
Tabla 2.- Lista de las 35 especies más ubicuas ordenadas de mayor a menor, según el número de cuadrículas UTM de $10 \mathrm{~km}$ de lado en las que se encuentra cada una de estas especies. Datos de García-Barros et al. (2004).

Table 2.- List of the 35 more ubiquitous species ordered by decreasing number of $10 \mathrm{~km}$ squares occupied. Data from GarcíaBarros et al. (2004).

\begin{tabular}{|c|c|c|c|}
\hline Especie & $\mathrm{N}^{\mathbf{0}} \mathrm{CUTM}$ & Especie & $\mathrm{N}^{\mathbf{0}} \mathrm{CUTM}$ \\
\hline Pieris rapae (Linnaeus, 1758) & 2442 & Pyronia tithonus (Linnaeus, 1771) & 1210 \\
\hline Colias crocea (Geoffroy, 1785 in Fourcroy) & 2253 & Pyronia cecilia (Vallantin, 1894) & 1198 \\
\hline Maniola jurtina (Linnaeus, 1758) & 1926 & Gonepteryx cleopatra (Linnaeus, 1767) & 1182 \\
\hline Pieris brassicae (Linnaeus, 1758) & 1811 & Lampides boeticus (Linnaeus, 1767) & 1160 \\
\hline Polyommatus icarus (Rottemburg, 1775) & 1810 & Hipparchia statilinus (Hufnagel, 1766) & 1129 \\
\hline Lasiommata megera (Linnaeus, 1767) & 1753 & Melanargia lachesis (Hübner, 1790) & 1127 \\
\hline Iphiclides podalirius (Linnaeus, 1758) & 1682 & Zerynthia rumina (Linnaeus, 1758) & 1121 \\
\hline Pararge aegeria (Linnaeus, 1758) & 1674 & Celastrina argiolus (Linnaeus, 1758) & 1092 \\
\hline Coenonympha pamphilus (Linnaeus, 1758) & 1638 & Gonepteryx rhamni (Linnaeus, 1758) & 1079 \\
\hline Vanessa cardui (Linnaeus, 1758) & 1623 & Callophrys rubi (Linnaeus, 1758) & 1076 \\
\hline Pontia daplidice (Linnaeus, 1758) & 1598 & Issoria lathonia (Linnaeus, 1758) & 1068 \\
\hline Lycaena phlaeas (Linnaeus, 1761) & 1526 & Melitaea phoebe (Denis \& Schiffermüller, 1775) & 1065 \\
\hline Papilio machaon (Linnaeus, 1758) & 1519 & Thymelicus sylvestris (Poda, 1761) & 1061 \\
\hline Euchloe crameri Butler, 1869 & 1353 & Pieris napi (Linnaeus, 1758) & 1048 \\
\hline Aricia cramera Eschscholtz, 1821 & 1279 & Pyronia bathseba (Fabricius, 1793) & 1046 \\
\hline Vanessa atalanta (Linnaeus, 1758) & 1261 & Thymelicus acteon (Rottemburg, 1775) & 1042 \\
\hline Leptotes pirithous (Linnaeus, 1767) & 1252 & Brintesia circe (Fabricius, 1775) & 1013 \\
\hline Leptidea sinapis (Linnaeus, 1758) & 1214 & & \\
\hline
\end{tabular}

captura, colector y localidad; y 9- el período (período de tiempo comprendido entre el primer y último año de datos de captura).

Se realizó una selección intuitiva de 25 cuadrículas UTM de 10 x $10 \mathrm{~km}$ situadas en diferentes puntos de la Península Ibérica (Figura 1) que presumimos perfectamente conocidas. Esta selección (Tabla 1) se basa en que son localidades clásicas en los muestreos lepidopterológicos, se llevan prospectando desde hace bastante tiempo, o han sido objeto de estudios intensivos debidamente documentados, por lo que se puede presumir que sus inventarios son, como promedio, completos y fiables.

Las listas de datos de estas cuadrículas (con un total de 21.093 registros) se aleatorizaron 5 veces. Se calculó en cada submuestra el valor acumulado de una serie de variables (que hemos denominado estimadores indirectos) para porcentajes acumulados de especies (sobre el total citado de cada cuadrícula) del $20 \%, 40 \%, 60 \%, 80 \%$ y $100 \%$. Los estimadores indirectos fueron los descritos en el apartado anterior. Los totales acumulados de las cinco submuestras de cada estimador para cada porcentaje acumulado de especies se promediaron, y el valor se transformó logarítmicamente. En otras palabras, se estimó el número de colectores, fechas, localidades, etc., que serían necesarios para registrar los diferentes porcentajes de especies de cada cuadrícula.

Mediante un análisis de regresión múltiple por etapas hacia delante (Forward Stepwise) (SPSS, 2003), se calculó la contribución de cada variable al porcentaje de especies acumulado. En un paso posterior, se empleó esta función para predecir el porcentaje de especies acumulado en el resto de las cuadrículas de $10 \mathrm{~km}$ de lado, usando sus datos reales de los estimadores seleccionados.

2. Criterios mínimos propuestos. La idea es establecer un criterio de mínimos, es decir, separar las cuadrículas que podrían contener datos válidos de las que, según un lepidopterólogo poco exigente, no cumplirían el mínimo de requisitos razonables. Se diseñaron para ello criterios basados en los valores totales para cada cuadrícula de ciertas variables, que en general caracterizarían una cuadrícula "razonablemente conocida", de acuerdo con la experiencia de los autores. Las variables usadas de la base de datos ATLAMAR1 fueron: número de especies, de fechas de captura (como número de meses) y de colectores, para estimar las cuadrículas que parecen estar mínimamente estudiadas. Se esta- 
blecieron tres combinaciones de criterios, siguiendo una gradación de menor a mayor exigencia, como sigue:

a) Cuadrículas de 10 x $10 \mathrm{~km}$ :

Categoría I: Con muestreos en tres o más meses comprendidos entre abril y agosto, con un mínimo de quince especies, con una representación de al menos diez especies entre una lista de las treinta y cinco especies más extendidas en el área de estudio (Tabla 2), y visitadas cuando menos por dos colectores diferentes. Es el conjunto de criterios menos restrictivo; es muy improbable que cualquier cuadrícula que no cumpla estos mínimos pueda haber sido prospectada en profundidad.

Categoría II: Hay evidencias de muestreo en cuatro o más meses comprendidos entre marzo y septiembre. Se han citado al menos 20 especies, la lista de especies incluye cuando menos diecisiete de las recogidas en la Tabla 2, y han sido visitadas por tres o más colectores.

b) Cuadrículas de 50 x $50 \mathrm{~km}$ :

Se probaron igualmente dos conjuntos de criterios adaptados a estas cuadrículas con dos niveles de exigencia, pero la diferencia entre ambos resultados fue tan escasa, que se optó por mantener únicamente el conjunto de condiciones correspondiente a la menos exigente de las dos (Categoría III): Hay datos fechados de tres o más meses entre abril y agosto, se han encontrado un mínimo de veinte especies, al menos diez de las especies ubicuas (Tabla 2) están representadas y existe constancia de la visita de más de dos colectores diferentes.

3. Curvas de acumulación. La forma de una curva de acumulación puede variar en función del orden de entrada de los datos, por lo que se aleatorizó ésta 100 veces mediante el programa EstimateS (Colwell, 2000). En consecuencia con los resultados descritos más adelante, se empleó el número de registros como unidad de esfuerzo, y se ajustaron los datos a la ecuación de Clench (Soberón \& Llorente, 1993; Jiménez-Valverde y Hortal, 2003; Hortal \& Lobo, 2002; Lobo \& Martín-Piera, 2002), de fórmula

$$
\mathrm{S}_{\mathrm{n}}=(\mathrm{a} * \mathrm{n}) /\left[1+\left(\mathrm{b}^{*} \mathrm{n}\right)\right]
$$

donde $\mathrm{S}_{\mathrm{n}}=$ número de especies, $\mathrm{a}=$ tasa de incremento de nuevas especies al principio del muestreo, $\mathrm{b}=$ parámetro relacionado con la forma de la curva, $\mathrm{y} \mathrm{n}=$ esfuerzo de muestreo.
Los parámetros de la función se ajustaron a los datos aleatorizados de cada curva mediante estimación no lineal utilizando el método de Simplex \& Quasi-Newton del paquete informático STATISTICA (StatSoft, 2004). La pendiente de la curva de acumulación en cada punto $\left(\right.$ Pendiente $\left.=\mathrm{a} /(1+\mathrm{bn})^{2}\right)$ determina la tasa de acumulación de especies, y decrece al aumentar el esfuerzo de muestreo, aproximándose a cero a medida que el inventario se acerca a la completitud. Se ha escogido un valor arbitrario de pendiente como punto de corte, un porcentaje estimado de fauna inventariada del 70\% o mayor, que parece ser razonable para muestras entomológicas (Jiménez-Valverde \& Hortal, 2003; Hortal \& Lobo, datos inéditos).

\section{Resultados}

ELECCIÓN DE LA UNIDAD DE ESFUERZO DE MUESTREO. El número de registros resultó ser la variable individual mejor correlacionada con el número acumulado de especies (Ver Tabla 3). En consecuencia, esta variable (de cálculo inmediato) se empleó como medida del esfuerzo de muestreo en los siguientes apartados del estudio.

\section{ESTUDIO DE LA CALIDAD DE LOS INVENTARIOS}

1. Estimadores indirectos. La ecuación de regresión obtenida a partir de los datos de cuadrículas bien muestreadas $\left(\mathrm{R}^{2}=0,889, \mathrm{p}<0,0001\right)$, sería de la siguiente forma:

Porcentaje de especies $=-91,59+78,69 x_{1}-12,02 x_{2}$ $+29,52 \mathrm{x}_{3}-17,59 \mathrm{x}_{4}+27,72 \mathrm{x}_{5}-18,52 \mathrm{x}_{6}-11,87 \mathrm{x}_{7}$

Siendo las variables el logaritmo decimal de: $\mathrm{x}_{1}$ : número de registros.

$\mathrm{x}_{2}$ : número de combinaciones diferentes de localidad, colector y fecha de captura.

$\mathrm{x}_{3}$ : período de tiempo comprendido entre el primer y último año de los que se poseen datos de captura en esa cuadrícula.

$\mathrm{x}_{4}$ : número de fuentes de información.

$\mathrm{x}_{5}$ : número de meses con muestras.

$\mathrm{x}_{6}$ : número de ejemplares.

$\mathrm{x}_{7}$ : número de localidades.

En la tabla 3 se presentan los datos de la regresión del porcentaje de especies sobre estas variables. Aplicando esta fórmula de regresión a todas las cuadrículas de $10 \mathrm{~km}$ de lado de las que se poseen datos, se obtienen 2897 cuadrículas con menos del $40 \%$ de su fauna inventariada, 592 que poseen 
Tabla 3.- Correlaciones univariantes y grado de significación de cada una de las variables obtenidas para el modelo seleccionado de regresión múltiple $\left(\mathrm{R}^{2}=0,889, \mathrm{p}<0,0001\right)$. La variable dependiente es el porcentaje de especies.

Table 3.- Univariate correlations and level of significance of each variable obtained by multiple regression $\left(\mathrm{R}^{2}=0.889\right.$, $\mathrm{p}<0.0001)$. The dependent variable is the percentage of species.

\begin{tabular}{lcl}
\hline Variables & R & p \\
\hline REGISTRO $\left(\mathrm{x}_{1}\right)$ & 0,887 & $\mathrm{p}<0,0001$ \\
EJEMPLARES $\left(\mathrm{x}_{6}\right)$ & 0,813 & $\mathrm{p}<0,001$ \\
FUENTE $\left(\mathrm{x}_{4}\right)$ & 0,601 & $\mathrm{p}<0,01$ \\
$\operatorname{LOCALIDAD}\left(\mathrm{x}_{7}\right)$ & 0,484 & $\mathrm{p}<0,011$ \\
$\operatorname{COMBINACION}\left(\mathrm{x}_{2}\right)$ & 0,480 & $\mathrm{p}<0,0001$ \\
MES $\left(\mathrm{x}_{5}\right)$ & 0,559 & $\mathrm{p}<0,001$ \\
PERIODO $\left(\mathrm{x}_{3}\right)$ & 0,376 & $\mathrm{p}<0,0001$ \\
\hline
\end{tabular}

entre un $40 \%$ y un $70 \%$, y 625 cuadrículas con un valor observado de al menos el $70 \%$ de su fauna estimada (Figura 2). Adicionalmente se pueden estimar 371 cuadrículas con más del 90\% de su fauna censada.

2. Criterios mínimos propuestos. Se observan 1075 cuadrículas UTM de $10 \mathrm{~km}$ de lado que cumplen los criterios de la categoría I, y 730 los de la categoría II (Figura 3). Del mismo modo, se obtienen 228 cuadrículas UTM de $50 \mathrm{~km}$ de lado mínimamente estudiadas (categoría III, Figura 4).

3. Curvas de acumulación. Mediante la aplicación de curvas de acumulación se obtiene un total de 180 cuadrículas UTM de $50 \mathrm{~km}$ de lado con un porcentaje estimado de fauna conocida igual o superior al $70 \%$. 96 de las cuadrículas de esta rejilla alcanzan niveles estimados superiores al 90\% (Figura 5).

RESUlTADOS COINCIDENTES. Se obtiene un total de 435 cuadrículas de $10 \mathrm{~km}$ de lado (Figura 6) que al coincidir en los resultados de todos los análisis aplicados (criterios mínimos propuestos de la categoría I y II, y regresión múltiple), pueden ser consideradas razonablemente bien inventariadas.

175 cuadrículas de $50 \mathrm{~km}$ de lado (Figura 7) son favorablemente evaluadas tanto mediante la aplicación de los criterios mínimos propuestos como mediante curvas de acumulación. Se comparó el grado de coincidencia entre las listas de cuadrículas de ambos procedimientos mediante un $\chi^{2}$, conclu- yéndose que un elevado porcentaje de cuadrículas son seleccionadas por ambos métodos $\left(\chi^{2}=84,648\right.$; $\mathrm{p}<0,0001)$, indicando que las muestras no difieren significativamente.

\section{Discusión}

La localización de áreas de alta concentración de diversidad requiere un adecuado cartografiado de la distribución geográfica detallada de las especies de mariposas (van Swaay \& Warren, 1999). La carencia de este conocimiento dificulta el diseño de una legislación eficaz relativa a la protección de especies y hábitats, así como la aplicación de la existente. A pesar de todos los esfuerzos realizados para la recolección de datos faunísticos de mariposas, actualmente persisten amplias áreas insuficientemente estudiadas, lo que lleva a realizar de forma cautelosa la comparación de fauna entre las diferentes localidades. Los resultados obtenidos están limitados debido a que la información faunística de la que disponemos es en muchos casos sesgada y heterogénea, pero pueden servir como indicativo del esfuerzo de muestreo necesario en zonas insuficientemente estudiadas. De hecho, constituyen cuando menos una primera aproximación objetiva de la distribución geográfica del esfuerzo faunístico efectivo relativo a estos insectos en la Península y Baleares, que permitirá plantear posteriores estudios sobre su biogeografía y conservación.

El número de registros es un buen indicador de la medida real de esfuerzo de muestreo, tal como se ha propuesto para otras bases de datos entomológicas (Hortal \& Lobo, 2002; Martín-Piera \& Lobo, 2003). Que esta medida sea preferible a, por ejemplo, el número de ejemplares (como podría esperarse) puede guardar relación con la heterogeneidad de la información, procedente de diversos orígenes a lo largo de un dilatado período de tiempo, así como con una tendencia a infravalorar notablemente el número real de capturas por la falta de información disponible en los datos. Un tipo de sesgo típico en bases de datos como la presente, viene dado por una parte de los datos bibliográficos en los que no se especifican indicaciones del esfuerzo de muestreo, como pueden ser fechas o números de ejemplares. De hecho, muchos registros no presentan el número de individuos capturados o avistados explícitamente. En nuestra base de datos se ha podido estimar un total de 416.454 ejemplares, número bastante bajo teniendo en cuenta el amplio período de tiempo tratado (dos siglos). En ocasiones, un intenso trabajo de campo queda reflejado en 


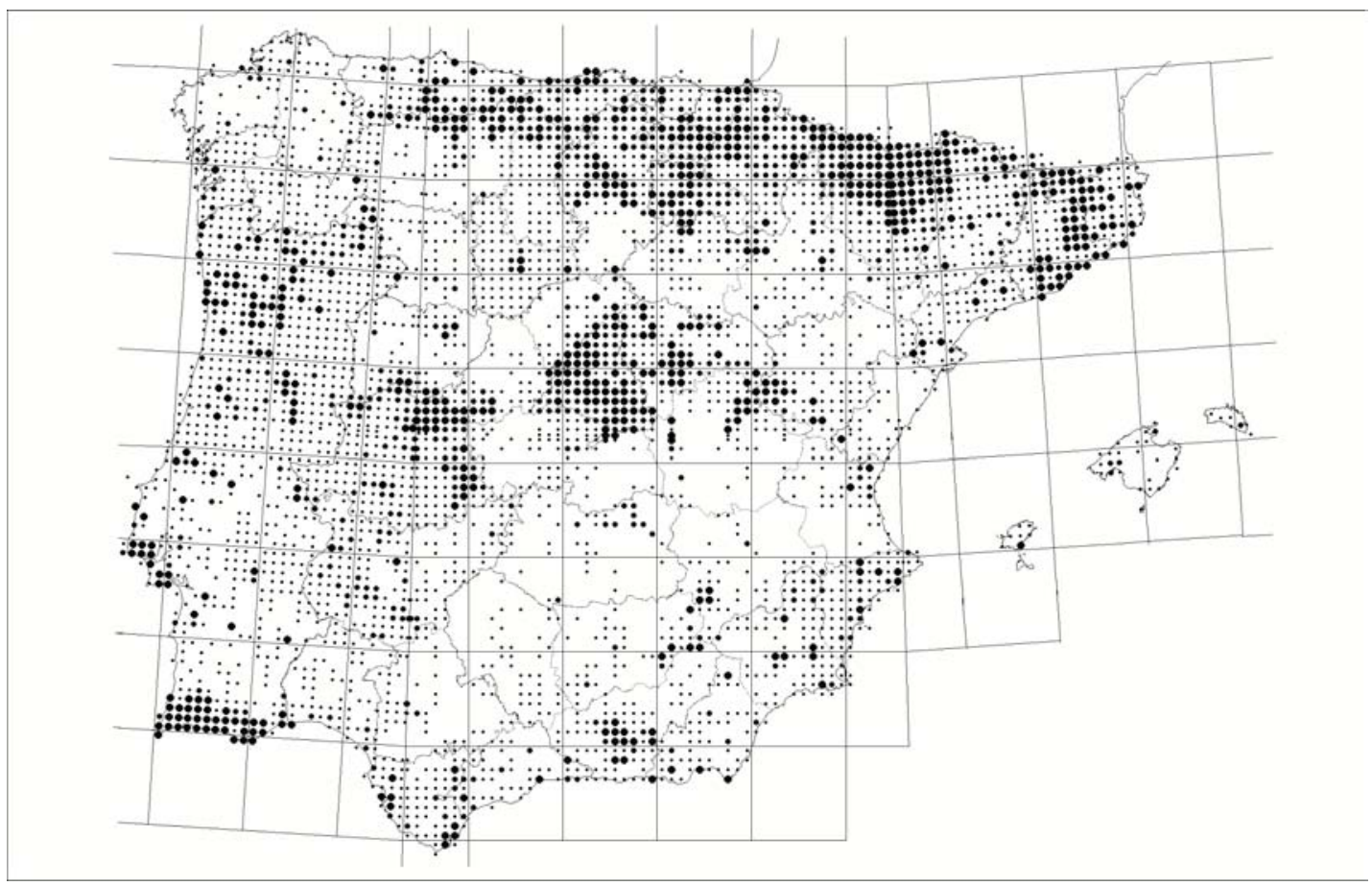

Fig. 2.- Esfuerzo de muestreo estimado. Distribución del esfuerzo relativo, estimado por regresión múltiple, en cuadrículas UTM de $10 \mathrm{~km}$ de lado. Se muestra una gradación del porcentaje estimado de fauna conocida en cada cuadrícula, mediante puntos de diferente tamaño. Puntos grandes indican cuadrículas con más del $70 \%$ de la fauna inventariada, puntos medianos entre el 40 y $70 \%$, y puntos pequeños menos del $40 \%$. Las áreas sin puntos carecen de datos.

Fig. 2.- Estimated sampling effort. Distribution of relative sampling effort (results from multiple regression approach) referred to $10 \times 10 \mathrm{~km}$ UTM squares. The estimated percentages are summarised with dots of different sizes: Large dots denote squares with more than $70 \%$ of their fauna recorded, medium dots indicate squares with percentages between 40 and $70 \%$, and small dots reveal squares with percentages of estimated relative effort lower than $40 \%$. Blank areas indicate lack of data.

una lista faunística. Un ejemplo es la cuadrícula de $10 \mathrm{~km}$ de lado 29SQA29, correspondiente al Palacio de Doñana. Esta cuadrícula está probablemente bien estudiada debido a que se enclava dentro de un Parque Nacional, pero los datos disponibles de esta cuadrícula son bibliográficos, la mayoría no presentan datos precisos de fecha de captura y número de ejemplares, y hay solamente 6 referencias documentadas (p. ej. Fernández Haeger et al., 1976; Venero, 1981; Rodríguez, 1991; Fernández Haeger, com. pers.), por lo que representan un número bastante pequeño de registros en nuestra base de datos.

Los métodos potencialmente aplicables a la estimación de intensidades de esfuerzo o a la detección de la diversidad potencial de muestras incompletas son variados, con diversos argumentos a favor del uso de curvas de acumulación (Soberón \&
Llorente, 1993; Hortal \& Lobo, 2002; JiménezValverde \& Hortal, 2003). Es interesante que una aproximación en principio burda, como la basada en criterios mínimos, muestre un elevado grado de coincidencia con la basada en, por ejemplo, curvas de acumulación (como en el presente caso, a escala de 50 x $50 \mathrm{~km}$ ). Este método carece de fundamento teórico, pero tiene uno de índole práctica, basado en este caso en la experiencia de los lepidopterólogos.

El estudio de la calidad de los inventarios basado en la elección de una serie de cuadrículas bien conocidas, reveló que sólo el 9,8\% de las cuadrículas de $10 \mathrm{~km}$ de lado de nuestro área de estudio se pueden considerar bien estudiadas (con más del $70 \%$ de su fauna censada, Figura 2). Es incluso posible que esta sea una perspectiva optimista, ya que únicamente el $6,8 \%$ del total reúnen además 


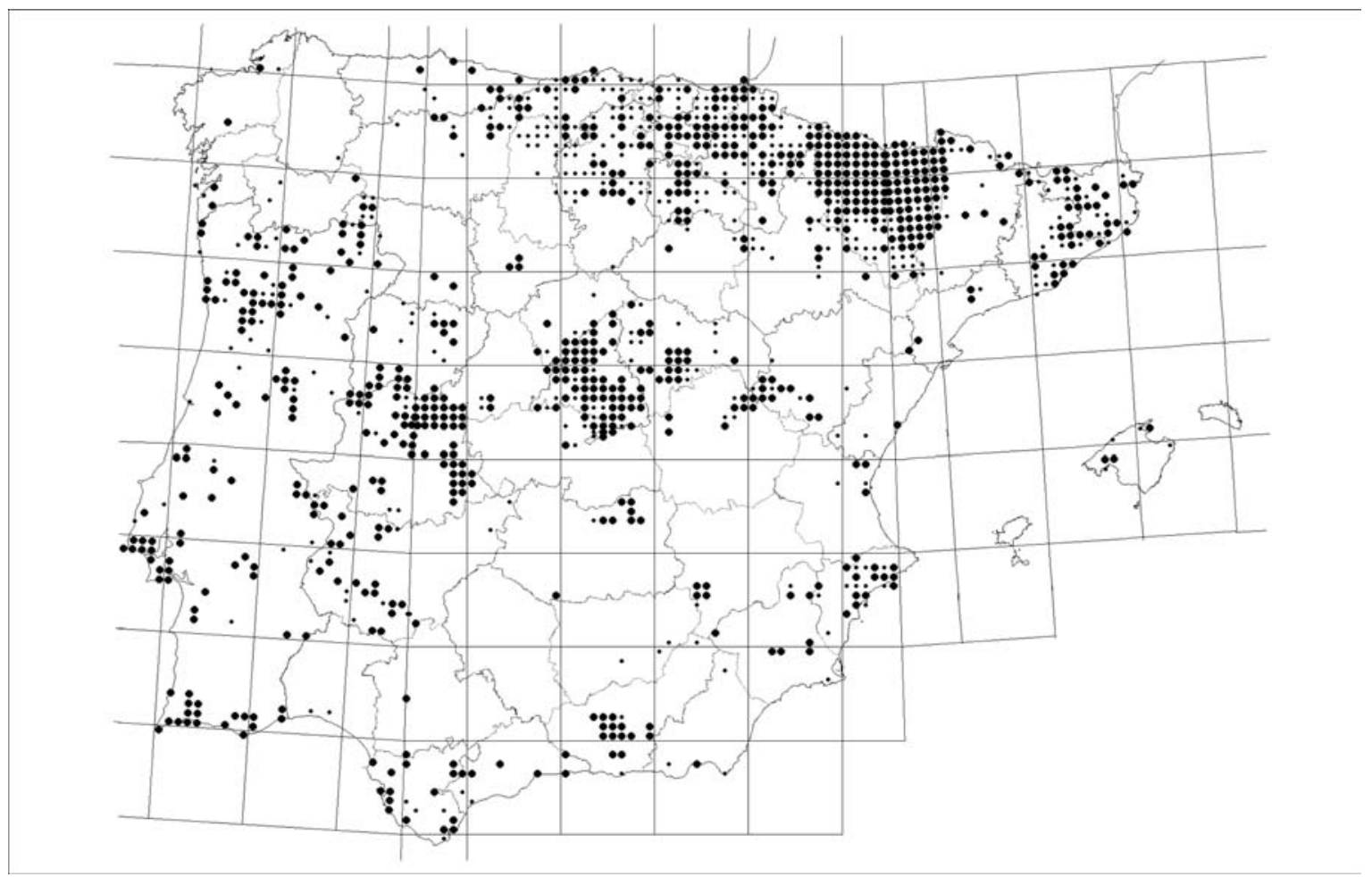

Fig. 3.- Distribución geográfica de la densidad de esfuerzo estimada en la Península Ibérica y Baleares, referida a cuadrículas UTM de $10 \mathrm{~km}$, estimada mediante la aplicación de criterios mínimos. Los puntos grandes representan las cuadrículas que cumplen la categoría I y II, y los pequeños las que cumplen la categoría I (ver texto).

Fig. 3.- Geographic distribution of the density of sampling effort in the Iberian Peninsula and Balearic Islands (10 x $10 \mathrm{~km}$ UTM grid), measured after criteria of minimal conditions. Large dots represent squares included in categories I and II, and small dots those included in category I (see text for details).

los requisitos de los conjuntos de criterios I y II. Para un $9,3 \%$ adicional, el nivel de conocimiento es claramente mejorable, y un $45,3 \%$ se encuentran definitivamente mal estudiadas (a las que habría que sumar todas aquellas cuadrículas de las que no se poseen datos, y que representan el 35,6\% del total). En cualquier caso, como mucho el $17 \%$ de las unidades de esta escala cumpliría el mínimo de requisitos razonables (Figura 3). Por tanto, el conocimiento faunístico de las mariposas ibéricas a una escala de cierto detalle (al menos cuadrículas de $10 \times 10 \mathrm{~km}$ ) es francamente deficitario, si bien se observa que, en rasgos generales, las áreas consideradas más ricas en especies de la Península Ibérica (Romo, inéd.) aparecen en nuestros análisis como bien muestreadas. Parece que existe una correlación entre las áreas mejor estudiadas y una mayor riqueza de especies (García-Barros et al.,
2000). Hasta hace poco, los lepidopterólogos visitaban con más frecuencia las áreas en las que su gran diversidad de especies había sido documentada con anterioridad. Sería por tanto interesante determinar hasta qué punto el esfuerzo de muestreo ha determinado la diversidad aparente o el patrón de riqueza estimada ha condicionado el patrón de conducta de los lepidopterólogos. El perfeccionamiento de la base de datos, con respecto a las empleadas en estimaciones preliminares (García-Barros \& Munguira, 1999), mejora los resultados para algunas regiones. Por ejemplo, la zona de Galicia que linda con Portugal, Murcia o Cataluña, que sin embargo distan aún bastante de poder considerarse bien estudiadas. Los resultados confirman, en cualquier caso, la existencia de amplias zonas de la Península carentes de datos casi por completo, y la necesidad de ahondar más 


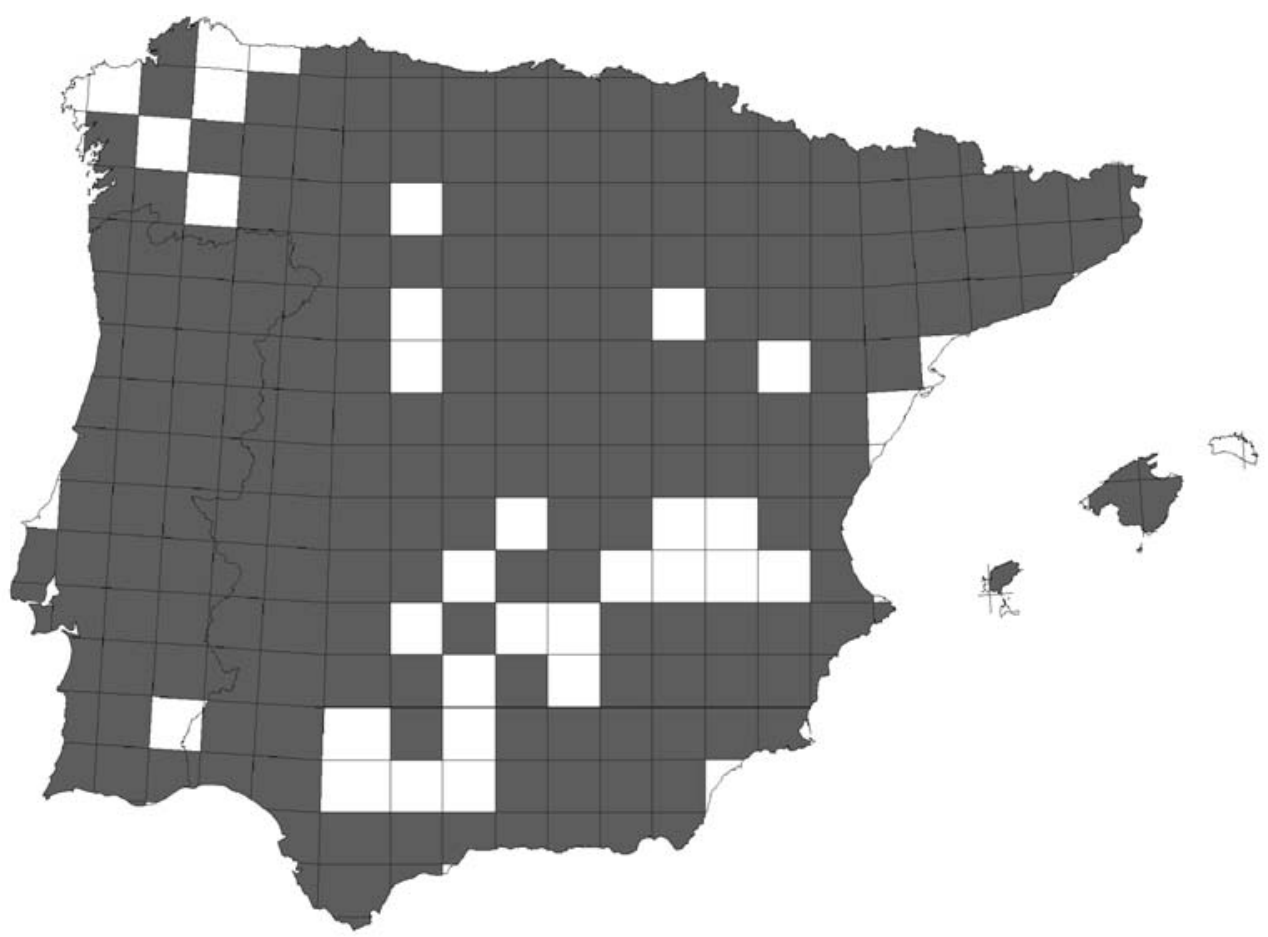

Fig. 4.- Distribución geográfica de la densidad de esfuerzo estimada en la Península Ibérica, en cuadrículas de 50 km. Las cuadrículas marcadas en oscuro son las que cumplen un mínimo de requisitos (categoría III, ver texto), y podrían por lo tanto ser consideradas para una estimación más detallada.

Fig. 4.- Geographic distribution of the density of sampling effort estimated in the study area, $50 \times 50 \mathrm{~km}$ UTM grid. Filled squares are those fulfilling only a minimum number of conditions (criteria of minimal conditions, category III, see text), and could therefore be considered for further analysis.

profundamente en sucesivos muestreos para completar los inventarios de las zonas menos favorecidas. La obtención de una cobertura amplia e intensa del terreno ibérico a escala de $10 \times 10 \mathrm{~km}$, requiere un notable incremento en el esfuerzo de muestreo. Este resultado es preocupante, ya que desde un punto de vista práctico (por ejemplo, de cara a evaluación de áreas para la conservación) es necesario un nivel de detalle geográfico incluso mayor que esta escala. Sería interesante revisar en detalle la tendencia actual del crecimiento de los datos faunísticos sobre este grupo, que no resultó muy prometedora en una estimación preliminar (García-Barros \& Munguira, 1999; García-Barros et al., 2000). Probablemente, sería necesario designar una red de cuadrículas de especial interés bien repartidas por todo el territorio, para su estudio exhaustivo. A esta escala, los puntos mejor estudiados se concentran en la zona más septentrional de la Península Ibérica, desde los Pirineos al
Sistema Cantábrico, en Madrid y áreas montañosas del centro peninsular, en el sistema Penibético y en la zona Norte, Lisboa y Algarve en Portugal (Figura 6). La baja proporción de cuadrículas satisfactorias, y su tendencia a la concentración geográfica, hacen poco recomendable esta escala para análisis de tipo general.

La cobertura faunística con una rejilla de mayor escala, la red de cuadrículas de $50 \mathrm{~km}$ de lado, es más satisfactoria. Más del $80 \%$ de estas cuadrículas cumplen un mínimo de requisitos (Figura 4), y el $64,1 \%$ del total puede considerarse suficientemente prospectado (Figura 5). Más aún, una buena proporción de estas cuadrículas (el 62,3\% del total, Figura 7) parecen especialmente fiables al cumplir simultáneamente los requisitos determinados de modo intuitivo y el nivel de saturación determinado por las curvas de acumulación. Por tanto, esta escala parece razonable para futuros estudios comparativos o de análisis biogeográfico de las mari- 


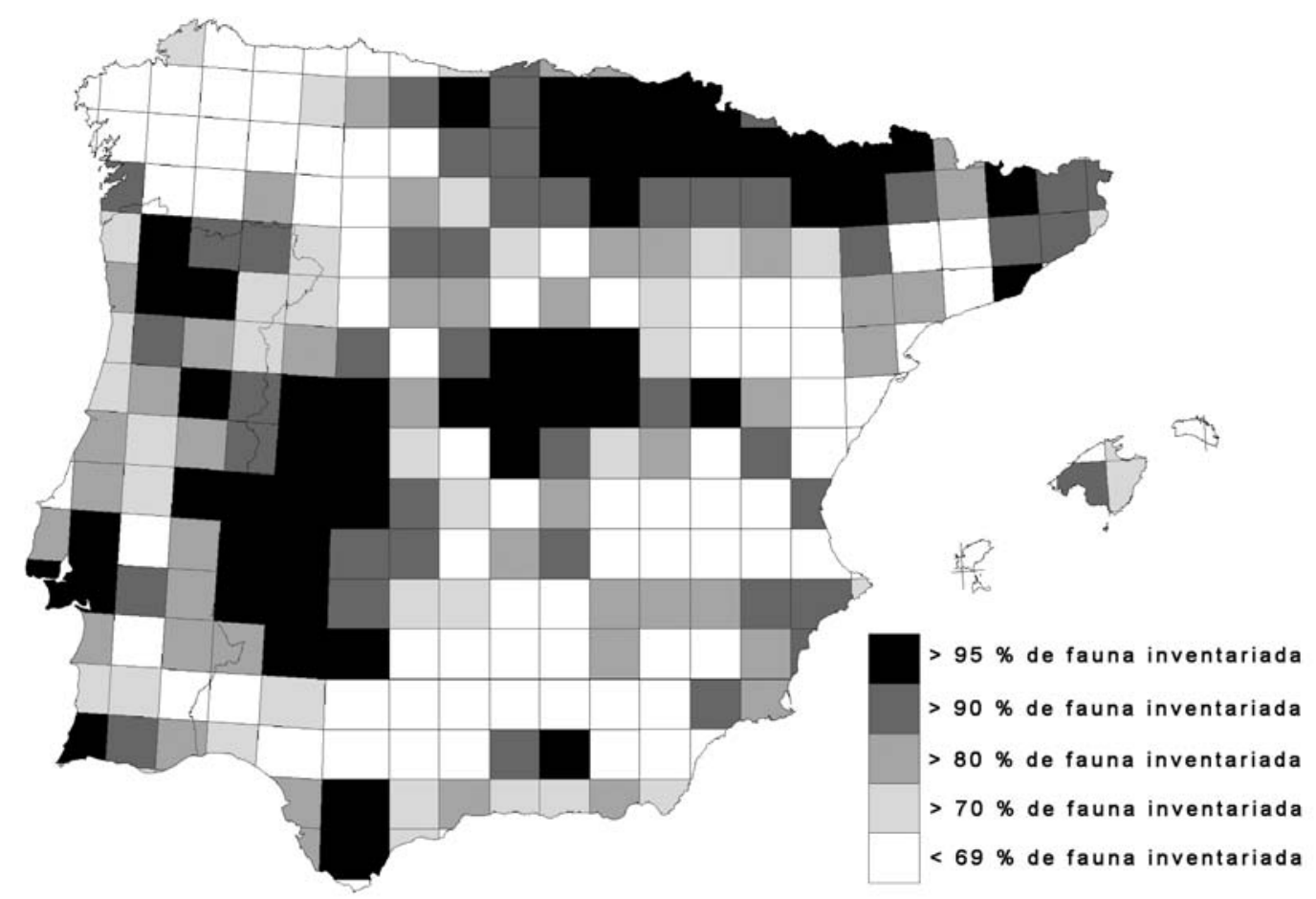

Fig. 5.- Cuadrículas UTM de $50 \mathrm{~km}$ de lado identificadas como bien muestreadas, mediante curvas de acumulación. Se representan en orden creciente cinco diferentes niveles de exigencia, mediante tonos de gris progresivamente más oscuros (saturación entre $70 \%$ al $95 \%$, además de las áreas pobres en datos o no muestreadas).

Fig. 5.- UTM $50 \mathrm{~km}$ squares identified as well sampled by means of accumulation curves. Five different levels of exigency are represented by increasing degrees of dark saturation (from $70 \%$ to $95 \%$, in addition to insufficiently known areas).

posas ibéricas, ya que proporciona un buen balance entre nivel de prospección y cobertura geográfica del área.

Se comprueba que la aplicación de los criterios mínimos propuestos selecciona un mayor número de cuadrículas respecto a los resultados obtenidos con las curvas de acumulación y la regresión múltiple. Esta sobreestimación no es preocupante, ya que con los criterios mínimos no estamos asumiendo cuadrículas bien muestreadas, sino zonas dentro de las cuales pueden encontrarse cuadrículas bien muestreadas, porque cumplen una serie de requisitos mínimos imprescindibles para considerarse bien estudiadas.

Como es lógico, el nivel de prospección estimado a ambas escalas (por ejemplo Figura 2 y Figura 5) está íntimamente relacionado (las cuadrículas grandes bien estudiadas suelen contener cuadrículas menores con inventarios relativamente completos). Como patrón general, las áreas favorablemente evaluadas se reparten por el Norte peninsular cantabro-pirenaico, en provincias como Barcelona, Madrid, Cádiz, Extremadura, y el área de Lisboa en Portugal. Los motivos de este patrón posiblemente representan una interacción entre dos factores, la densidad de población humana (y su relación con las vías de acceso a regiones naturales) y la riqueza de especies (Dennis \& Thomas, 2000). Por ejemplo, buen número de localidades bien estudiadas se concentran alrededor de Madrid y Barcelona, al igual que en Lisboa, grandes ciudades cuya fauna lepidopterológica ha sido prospectada concienzudamente, y por otra parte, presentan alta densidad de población humana, lo que favorece la existencia de más personas dedicadas al estudio de las mariposas (Heath et al., 1984; Dennis et al., 1999). El lugar de residencia de los lepidopterólogos también puede tener una elevada importancia en el grado de conocimiento de la diversidad de mariposas de una determinada zona (Martín \& Gurrea, 1999; Dennis et al., 1999). El caso de Extremadura responde a un elevado esfuerzo dedi- 


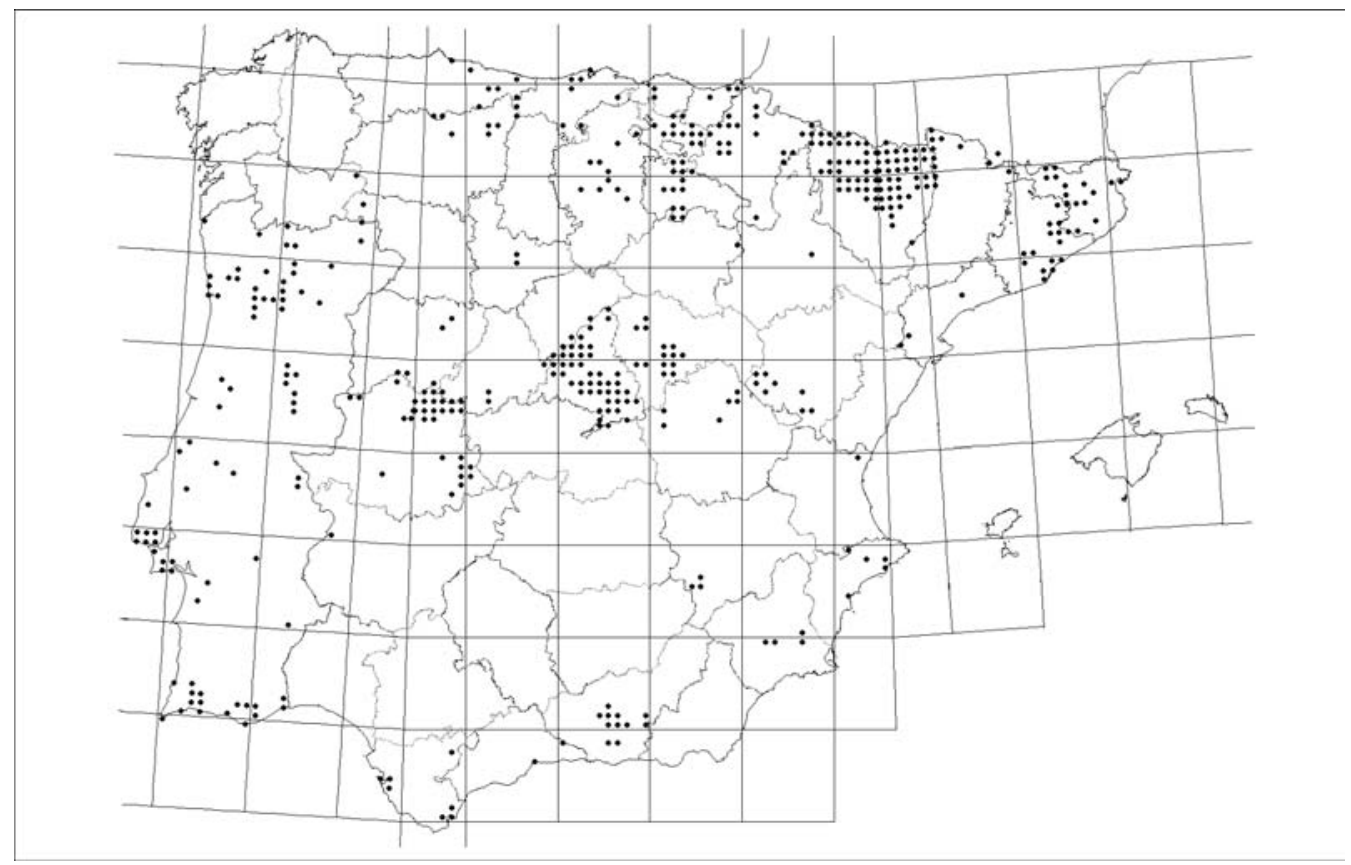

Fig. 6.- Cuadrículas fiables, rejilla de $10 \mathrm{~km}$. Distribución de las 435 cuadrículas UTM de $10 \mathrm{~km}$ de lado determinadas como bien muestreadas en todos los análisis efectuados (criterios mínimos de la categoría I y II, y regresión múltiple).

Fig. 6.- Reliable squares, $10 \mathrm{~km}$ grid. Distribution of the $43510 \mathrm{~km}$ UTM squares that were determined as well sampled in all analyses (criteria of minimal conditions of category I and II, and multiple regression).

cado a su estudio en tiempos recientes (GarcíaVillanueva et al., 1997; Blázquez et al., 2003). La zona de los Pirineos es una zona muy rica en especies y, por tanto, objetivo principal de muchos lepidopterólogos a la hora de planear sus muestreos. La distribución de esfuerzos parece interactuar con la riqueza de especies que, en la Península, se correlaciona con un gradiente SO-NE (el número de especies aumenta conforme disminuye la distancia a Pirineos), y con la altitud (es decir, las cadenas montañosas: Martín \& Gurrea, 1990).

\section{Conclusiones}

1) El uso de estimadores indirectos para estudiar la calidad de los inventarios, permitió identificar un total de 625 cuadrículas UTM de $10 \mathrm{~km}$ de lado bien inventariadas (un 9,8\% del total de cuadrículas de nuestro área de estudio). Otras 592 cuadrículas se encontrarían en una situación mejorable $(9,3 \%)$ y 2897 UTM están mal estudiadas $(45,3 \%)$. 2281 cuadrículas carecen de datos, constituyendo un $35,7 \%$.

2) La aplicación de criterios mínimos señaló un máximo de un $16,8 \%$ de cuadrículas de $10 \mathrm{~km}$ de lado bien inventariadas, que se elevó hasta un $81,1 \%$ en el caso de las cuadrículas de $50 \mathrm{~km}$.

3) La aplicación de curvas de acumulación produjo un total de 180 cuadrículas UTM de $50 \mathrm{~km}$ de lado con más del $70 \%$ de su fauna inventariada $(64,1 \%$ del total).

4) Si se comparan todos los diferentes análisis aplicados, se observan resultados coincidentes en un total de 435 cuadrículas de $10 \mathrm{~km}(6,8 \%)$, y 175 cuadrículas de $50 \mathrm{~km}(62,3 \%)$. Estas cuadrículas reúnen los requisitos más exigentes, distribuyéndose por parte de los Pirineos y Sistema Cantábrico, el Sistema Central y Penibético, y las regiones más occidentales de Portugal. 


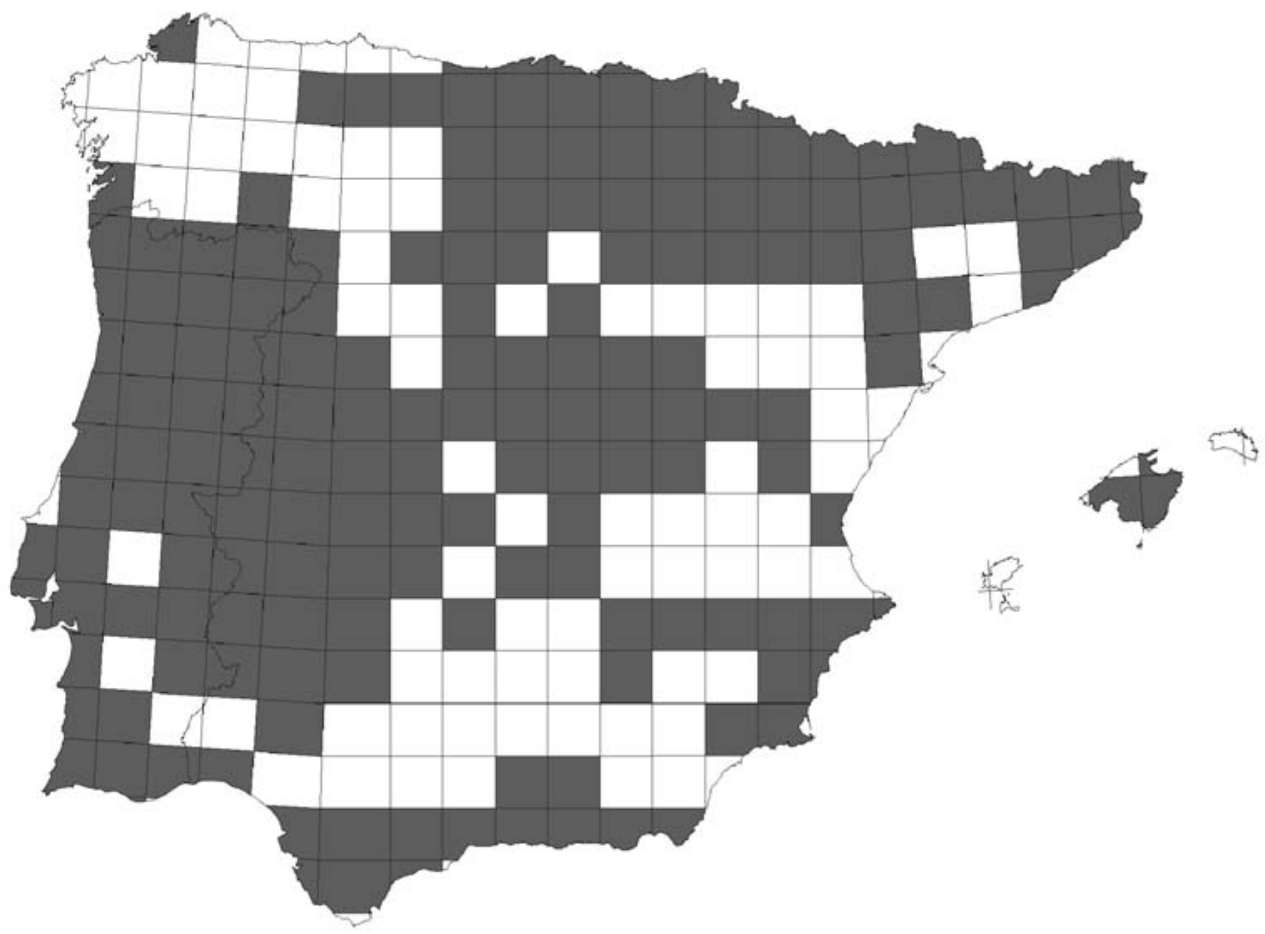

Fig. 7.- Cuadrículas fiables, rejilla de $50 \mathrm{~km}$. Distribución de las 175 cuadrículas UTM de $50 \mathrm{~km}$ de lado identificadas como bien conocidas en todos nuestros análisis (criterios mínimos de la categoría III, y curvas de acumulación).

Fig. 7.- Reliable squares, $50 \mathrm{~km}$ grid. Distribution of the 175 UTM $50 \mathrm{~km}$ squares identified as well sampled in all analyses (criteria of minimal conditions of category III, and accumulation curves).

5) A la vista del porcentaje relativamente bajo de cuadrículas de $10 \mathrm{~km}$ de lado razonablemente estudiadas a lo largo de un considerable período de tiempo, y considerando de forma realista el esfuerzo faunístico actual, no parece posible que se pueda completar en un plazo razonable de tiempo, de acuerdo con García-Barros \& Munguira (1999). Por ello, sería conveniente designar una red de espacios que sería necesario muestrear.

6) Las cuadrículas de $50 \mathrm{~km}$ representan la unidad de terreno menor que es razonable usar con propósitos comparativos para estudios posteriores de estos insectos en el ámbito ibero-balear.

\section{AGRADECIMIENTOS}

Este trabajo ha sido posible gracias al apoyo incondicional de varias personas, entre ellas caben destacar: Emilio, por su inestimable ayuda y apoyo; el Dr. Miguel López Munguira por sus sugerencias para mejorar este trabajo; el Dr. Jorge Miguel Lobo, que nos ayudó a introducirnos en el mundo de las curvas de acumulación; El resto de compañeros del laboratorio que de una manera $\mathrm{u}$ otra nos han ayudado a seguir adelante, $\mathrm{y}$ los miembros del proyecto ATLAMAR (referencia REN20000466 GLO), que han contribuido a formar la base de datos imprescindible para la realización de este trabajo.

Parte de este trabajo ha sido financiado por la beca F.P.U. referencia AP2002-0147 concedida por el Ministerio de Educación Cultura y Deporte a Helena Romo.

\section{Referencias}

Blázquez, A., Nieto, M. A. \& Hernández, J. L., 2003. Mariposas diurnas de la provincia de Cáceres. Junta de Extremadura, Consejería de Agricultura y Medio Ambiente. Mérida. 211 pp.

COLWELL, R. K., 2000. EstimateS: Statistical Estimation of Species Richness and Shared Species from Samples (Software and User's Guide). Versión 6.0b1. Disponible en http://viceroy.eeb.uconn.edu/estimates

Dennis, R. L. H., Sparks, T. H. \& Hardy, P. B., 1999. Bias in butterfly distribution maps: the effects of sampling effort. Journal of Insect Conservation, 3: 33-42. 
DENNIS, R.L.H. \& THOMAS, C.D., 2000. Bias in butterfly distribution maps: the influence of hot spots and access. Journal of Insect Conservation, 4: 73-77.

FERnÁndez Haeger, H., García, I. \& Aguilar, J., 1976. Guía de las mariposas de Doñana. Naturalia Hispanica, $\mathrm{n}^{\mathrm{o}}$ 6. Servicio de Publicaciones, Ministerio de Agricultura. Madrid. 55 pp.

García-Barros, E., Garcia-Pereira, P. \& Munguira, M. L., 2000. The geographic distribution and state of butterfly faunistic studies in Iberia (Lepidoptera Papilionoidea Hesperioidea). Belgian Journal of Entomology, 2: 111-124.

García-Barros, E. \& Munguira, M. L., 1999. Faunística de mariposas diurnas en España peninsular. Áreas poco estudiadas: una evaluación en el umbral del Siglo XXI (Lepidoptera: Papilionoidea \& Hesperiidae). SHILAP Revista de Lepidopterología, 27(106): 189-202.

García-Barros, E., Munguira, M. L., Martín, J., Romo, H., Garcia-Pereira, P. \& Maravalhas, E. S., 2004. Atlas de las mariposas diurnas de la Península Ibérica e islas Baleares (Lepidoptera: Papilionoidea \& Hesperioidea). Monografías S.E.A., vol. 11. Zaragoza. $228 \mathrm{pp}$.

Garcia-Pereira, P. N. C., 2003. Mariposas diurnas de Portugal continental: Bases para la conservación de la diversidad. Tesis doctoral. Universidad Autónoma de Madrid. 371 pp.

García-Villanueva, V., Blázquez, A., Novoa, J. M. \& NiETo, M. A., 1997. Atlas de los lepidópteros ropalóceros de Extremadura. Instituto Extremeño de Entomología. Badajoz. 122 pp.

Gotelli, N. J. \& Colwell, R. K., 2001. Quantifying biodiversity: procedures and pitfalls in the measurement and comparison of species richness. Ecology Letters, 4: 379-391.

Heath, J., Pollard, E. \& Thomas, J. A., 1984. Atlas of Butterflies in Britain and Ireland. Viking. London. $158 \mathrm{pp}$.

Hortal, J., Garcia-Pereira, P. \& García-Barros, E., 2004. Butterfly species richness in mainland Portugal: predictive models of geographic distribution patterns. Ecography, 27: 68-82.

HorTAL, J. \& LoBO, J. M., 2002. Una metodología para predecir la distribución espacial de la diversidad biológica. Ecología, 16: 405-432.

JimÉNEZ-VAlVERDE, A. \& HoRTAL, J., 2003. Las curvas de acumulación de especies y la necesidad de evaluar la calidad de los inventarios biológicos. Revista Ibérica de Aracnología, 8: 151-161.

KudrnA, O., 2002. The distribution atlas of European Butterflies. Oedippus, 20: 1-342.

Lobo, J. M. \& Martín-Piera, F., 2002. Searching for a Predictive Model for Species Richness of Iberian Dung Beetle Based on Spatial and Environmental Variables. Conservation Biology, 16: 158-173.
MapINFO, 1994. Mapinfo version 3.0.2. User manual. MapInfo Corporation. One Global View, Troy, New York.

Martín, J. \& Gurrea, P., 1990. The peninsular effect in Iberian butterflies (Lepidoptera: Papilionoidea and Hesperioidea). Journal of Biogeography, 17: 85-96.

Martín, J. \& Gurrea, P., 1999. Áreas de especiación en España y Portugal. Boletín de la Asocioción española de Entomología, 23(1-2): 83-103.

MArtín-Piera, F. \& Lobo, J. M., 2003. Database records as a sampling-effort surrogate to predict spatial distribution of insects in either poorly o unevenly surveyed areas. Acta Entomológica Ibérica e Macaronésica, 1: 23-35.

MunguiRA, M. L., 1995. Conservation of butterfly habitats and diversity in European Mediterranean countries. In: A. S. Pullin (ed.). Ecology and conservation of butterflies. Chapman \& Hall. London: 277-289

Myers, N., Mittermeier, R. A., MitTermeier, C. G., DA FonseCA, G. A. B. \& Kent, J., 2000. Biodiversity hotspots for conservation priorities. Nature, 403: 853-858.

New, T.R., 1991. Butterfly Conservation. Oxford University Press. Oxford. xi +224 pp.

Palomo, L. J. \& Antúnez, A., 1992. Los atlas de distribución de especies. In: J. M. Vargas, R. Real \& A. Antúnez (eds.). Objetivos y métodos biogeográficos. Aplicaciones en Herpetología. Monografías de Herpetología, 2: 39-50.

Rodríguez, J., 1991. Las mariposas del Parque Nacional de Doñana. Biología y Ecología de Cyaniris semiargus $y$ Plebejus argus. Tesis doctoral. Dpto. Biología Vegetal y Ecología. Universidad de Córdoba. 191 pp. (Inédita).

Soberón, J. \& Llorente, J., 1993. The use of species accumulation functions for the prediction of species richness. Conservation Biology, 7: 480-488.

SPSS, 2003. SPSS for Windows (Statistical Package for Social Sciences). Versión 12.0.1. SPSS Inc., Chicago. http://www.spss.com/

StATSOFt, InC., 2004. STATISTICA (data analysis software system), version 6. Tulsa. www.statsoft.com.

VAn SWAay, C. A. M. \& Warren, M.S., 1999. Red Data Book of European Butterflies (Rhopalocera). Nature and Environment, No. 99, Council of Europe Publishing. Strasbourg. 260 pp.

VENERO, J. L., 1981. Distribución estacional de los ropalóceros en la reserva biológica de Doñana. SHILAP Revista de Lepidopterología, 9(36): 279-284.
Recibido, 3-II-2005 Aceptado, 24-V-2005 Publicado, 14-VII-2005 